\section{Extranodal NK/T-Cell Lymphoma, Nasal Type, Presenting as Facial Cellulitis}

Received: October 09, 2018; Accepted: October 11, 2018; Published: October 14, 2018

\section{Clinical Image}

A 26-years-old young lady with no prior medical history, presented to the hospital with and swelling of the right cheek and submandibular area rapidly increasing in volume. The patient was previously diagnosed with sinus infection and discharged on antibiotics, but the symptoms did not improve. Two weeks later the patient was hospitalized, on examination the patient was febrile with temperature $38.5^{\circ} \mathrm{C}$, with an edema, tenderness, and warmth of the right cheek without discharge. A CT scan (Figure 1) was performed that showed a soft tissue and fat swelling in the masticator pterygomandibular and temporal space infiltrating masseter muscle with subcutaneous fat induration without collection, associated to a right maxillary sinusitis, and cervical lymphadenopathy. It concludes to a facial soft tissue cellulitis and was initiated on empiric intravenous antibiotics. The patient showed a worsening of skin erythema, right facial enema, with and appearance of ulcerated lesion on hard palate. A new CT scan (Figure 2) was performed showing a right paranasal and parapharyngeal mass infiltrating maxillary sinus, hard palate and mandibular bone, with nasopharyngeal posterior wall enlargement, and multiple bilateral cervical lymphadenopathies. The patient underwent a biopsy of hard palate (ulcerated lesion) and mandibular bone. The histopathology result showed a T-lymphocytic infiltration. Immunohistochemical studies showed malignant cells with CD2+, and CD3 (cytoplasmic)+, but CD20-,
Mouzount A*, Mosse W, Majjaoui SE, Kebdani T and Benjaafar $\mathrm{N}$

Department of Radiation Oncology, National Institute of Oncology, Mohammed V University, Rabat, Morocco

\section{*Corresponding author: Mouzount A \\ $\equiv$ ma.mouzount@gmail.com \\ Department of Radiation Oncology, National Institute of Oncology, Mohammed V University, Rabat, Morocco.}

Tel: +90 3123044683

Citation: Mouzount A, Mosse W, Majjaoui SE, Kebdani T, Benjaafar N (2018)

Extranodal NK/T-Cell Lymphoma, Nasal Type, Presenting as Facial Cellulitis. Arch Can Res Vol.6 No.3:i33.
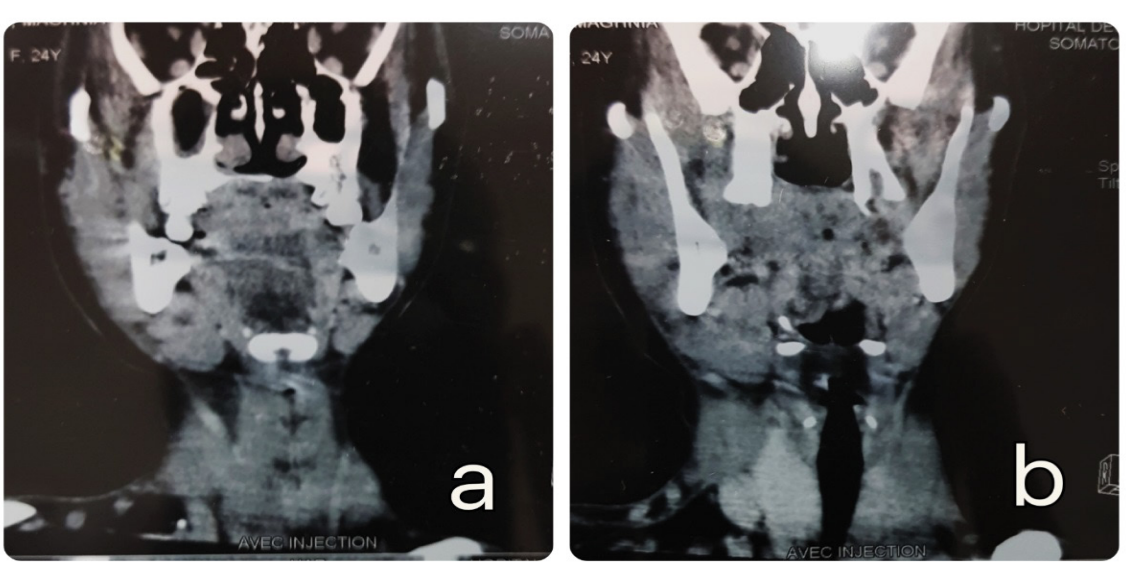

Figure 1 Coronal plane CT scan showing a swelling of the right masticator space infiltrating in filtrating masseter muscle with subcutaneous fat induration without collection. 


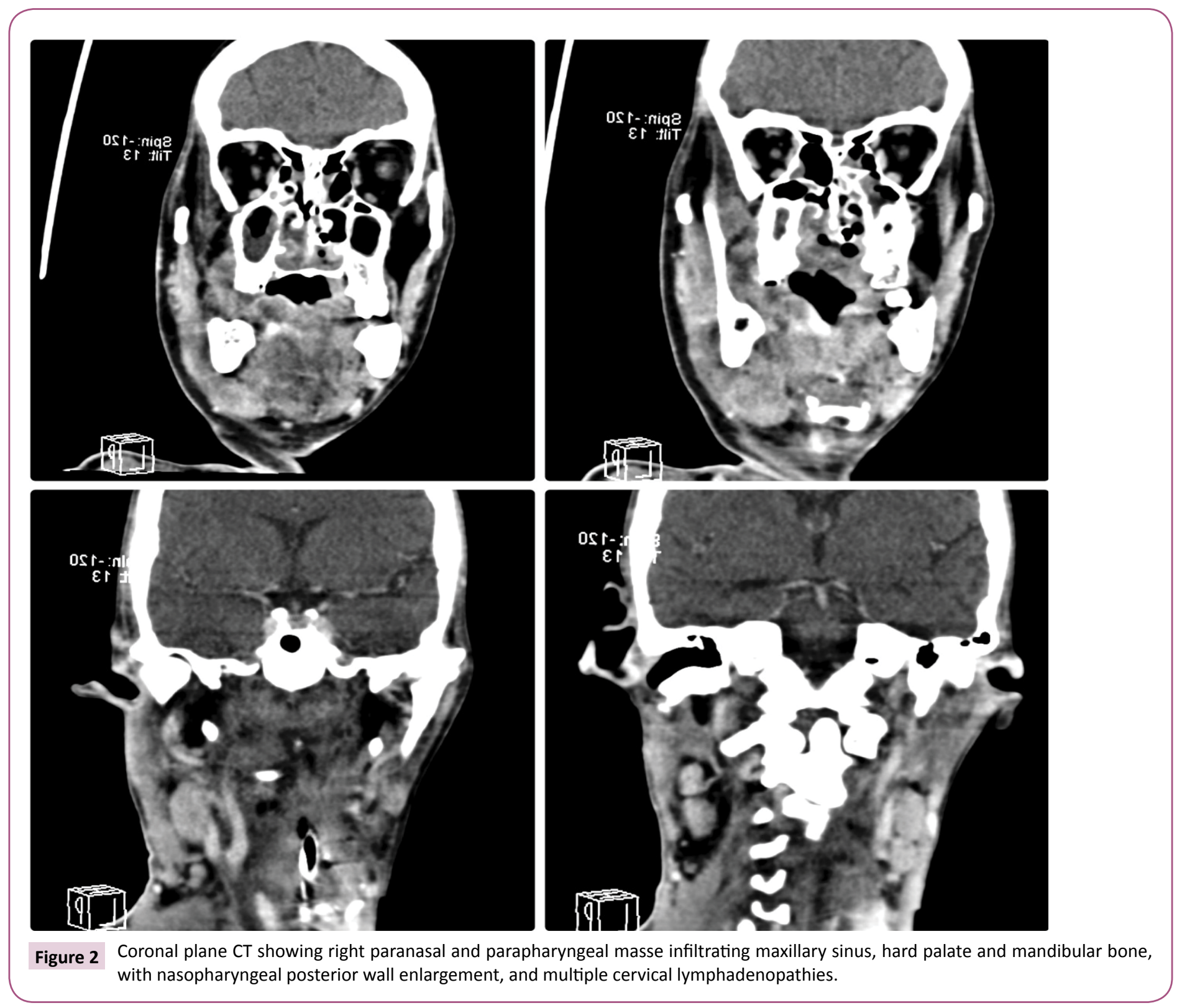

granzyme B+, CD56-. Ki67 proliferation index was about $80 \%$. In situ hybridization for Epstein-Barr virus (EBV) encoded RNA (EBER) showed positive reaction. These features were consistent with NK/T-Cell Lymphoma, Nasal type. CT scans of the chest and abdomen were normal. Bone marrow aspirate showed reactive hypercellular marrow with no obvious infiltration. No positron emission tomography (PET) scan was done. The patient had 4 cycle of CHOEP chemotherapy with good response after 3 cycle up to $80 \%$ shrinkage then referred to our department for sandwich radiotherapy. The patient is planned for external beam radiotherapy, then chemotherapy will be continued. 